\title{
Abrikosov vortex escape from a columnar defect as a topological electronic transition in vortex core
}

\author{
A. S. Mel'nikov and A. V. Samokhvalov \\ Institute for Physics of Microstructures, Russian Academy of Sciences, 603950 Nizhny Novgorod, GSP-105, Russia
}

(Dated: October 31, 2019)

\begin{abstract}
We study microscopic scenario of vortex escape from a columnar defect under the influence of a transport current. For defect radii smaller than the superconducting coherence length the depinning process is shown to be a consequence of two subsequent topological electronic transitions in a trapped vortex core. The first transition at a critical current $j_{L}$ is associated with the opening of Fermi surface segments corresponding to the creation of a vortex-antivortex pair bound to the defect. The second transition at a certain current $j_{d}>j_{L}$ is caused by merging of different Fermi surface segments, which accompanies the formation of a freely moving vortex.

PACS numbers:
\end{abstract}

The study of magnetic and transport properties of type-II superconductors in the presence of artificial pinning centers is known to be an important direction in the physics of vortex matter [1]. An obvious first step to understanding of the pinning related phenomena is associated with the evaluation of the individual pinning force acting on a vortex in the presence of a defect. Various approaches used for such analysis are based on the observation that a defect attracts the vortex in order to avoid loss of condensation energy in the core and to decrease a kinetic energy due to perturbations of screening currents and magnetic field induced by the defect [2-5]. Close to the critical temperature $T_{c}$ both these effects can be described within the Ginzburg-Landau (GL) theory valid for large length scales well exceeding the superconducting coherence length $\xi$ at zero temperature.

Such long wavelength approach is, of course, no more valid for low temperatures $T \ll T_{c}$ and small defect size $a \lesssim \xi$. Microscopic approach for calculation of the pinning energy and appropriate modification of the GL functional for a particular case of a small point impurity with the scattering cross section $\sigma_{s c} \ll \xi^{2}$ have been previously studied in Ref. [6, 7]. Considering the microscopic theory one should take into account the behavior of the subgap fermionic states bound to the vortex core which are known to determine both the structure and dynamics of vortex lines in the low temperature limit. These subgap states are known to form the so-called anomalous spectral branch crossing the Fermi level. For well separated vortices the behavior of the anomalous branches can be described by the Caroli-de Gennes-Matricon (CdGM) theory 8]: for each individual vortex the energy $\varepsilon_{0}(\mu)$ of subgap states varies from $-\Delta_{0}$ to $+\Delta_{0}$ as one changes the angular momentum $\mu$ defined with respect to the vortex axis. Here $\Delta_{0}$ is the superconducting gap value far from the vortex axis. At small energies $|\varepsilon| \ll \Delta_{0}$ the spectrum is a linear function of $\mu: \varepsilon_{0}\left(\mu, k_{\perp}\right) \simeq-\mu \hbar \omega$, where $\hbar \omega \approx \Delta_{0} \ln \Lambda /\left(k_{\perp} \xi\right)$ is the interlevel spacing, $\xi=\hbar V_{F} / \Delta_{0}, k_{\perp}^{2}=k_{F}^{2}-k_{z}^{2}$, $k_{z}$ is the momentum projection on the vortex axis, $k_{F}$ and $V_{F}$ are the Fermi momentum and velocity, respectively. The logarithmic factor with $\Lambda \sim \Delta_{0} / T$ appears at low temperatures due to the so-called Kramer-Pesch effect [9, 10]. Neglecting for $T \gg \hbar \omega$ the quantization of the angular momentum $\mu$ one can consider the anomalous branch to cross the Fermi level at $\mu=0$ for all orientations of the Fermi momentum $\mathbf{k}_{F}$. Thus, in the space $\mu-\mathbf{k}_{F}$ we obtain a Fermi surface (FS) for excitations localized within the vortex core (see [11] for review). Changing magnetic field or transport current we can get switching between different vortex states which is accompanied by the changes in the FS topology. Such topological transitions in quasiparticle spectra of vortex systems are similar to the well-known Lifshits transitions which occur in the band spectra of metals [12, 13]. One can separate two generic examples of such transitions in vortex matter: (i) opening of FS segments corresponding to the creation of vortex-antivortex pairs [14, 15]; (ii) merging and reconnection of different FS segments via the Landau-Zener tunneling [15, 16]. The basic properties of vortex matter such as pinning and transport characteristics, heat transport in the vortex state and peculiarities of the local density of states should be strongly affected by these changes in the FS topology. The goal of this Letter is to suggest a theory of topological electronic transitions which occur in a pinned vortex core under the influence of transport current.

The mechanism of these transitions is closely related to the effect of elastic scattering on the CdGM levels. Resulting modification of the anomalous spectral branch is noticeable even for impurity atoms introduced in a vortex core [17] and becomes much more pronounced provided we consider defects of the size well exceeding the Fermi wavelength. The absence of the FS is a hallmark of a vortex pinned by an insulating columnar defect and it is natural to expect that vortex depinning should be accompanied by the FS formation. For a vortex line trapped by such defect the subgap spectrum has been analyzed recently within the quasiclassical approach in Ref. [18]. For rather large angular momenta $|\mu|>\mu_{a}=k_{\perp} a$ quasiclas- 

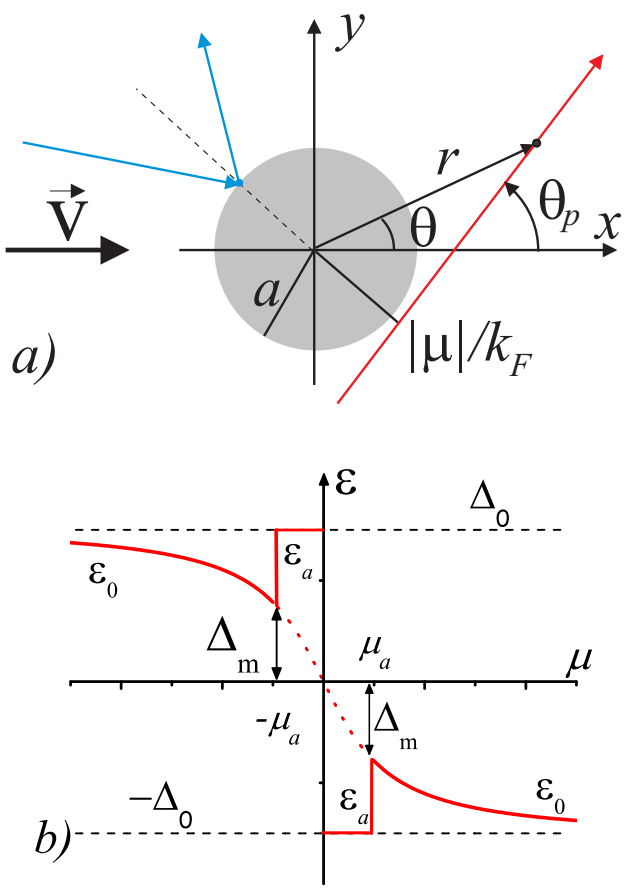

Figure 1: Fig.1 Different types of quasiclassical trajectories (a) and spectrum of subgap states (b) for a vortex pinned by the defect.

sical trajectories of electron-hole excitations do not experience reflection at the defect surface and, thus, the subgap spectrum coincides with the CdGM one. In the opposite case $|\mu| \leq \mu_{a}$ the normal reflection of trajectories at the defect destroys the low energy part of CdGM spectral branch: with the decrease in $|\mu|$ below the threshold value $\mu_{a}$ the energy of the subgap bound state rapidly approaches the superconducting gap $\Delta_{0}$ (see Fig. 1). Thus, contrary to the CdGM case the subgap spectral branch of the pinned vortex does not cross the Fermi level: there appears a minigap $\Delta_{m}(a) \sim\left|\varepsilon_{0}\left(k_{F} a, k_{F}\right)\right|$ in the quasiparticle spectrum. The spectral branch $\varepsilon_{a}$ for trajectories touching the defect can be approximated by vertical lines passing from $\pm \Delta_{m}$ to $\pm \Delta_{0}$ for $\mu=\mp \mu_{a}$ (see Fig. 1b).

In this Letter we propose a microscopic scenario of vortex escape from a columnar defect of a radius $a$ smaller than the coherence length $\xi$ under the influence of an external supercurrent $\mathbf{j}=e n_{s} \mathbf{V}$ applied perpendicular to the vortex axis (Fig.1). The transport supercurrent results in a Doppler shift $\varepsilon_{d}=(\hbar \mathbf{k} \cdot \mathbf{V})$ of the quasiparticle energy and the subgap spectrum takes the form:

$$
\tilde{\varepsilon}\left(\mu, k_{\perp}, \theta_{p}\right)=\varepsilon\left(\mu, k_{\perp}\right)+\hbar k_{\perp} V \cos \theta_{p},
$$

where $\varepsilon=-\Delta_{0} \operatorname{sign} \mu$ for quasiparticles with $|\mu|<\mu_{a}$ and $\varepsilon=\varepsilon_{0}$ for quasiparticles with $|\mu|>\mu_{a}$. Here the quasiparticle momentum $\mathbf{k}=k_{\perp}\left(\cos \theta_{p}, \sin \theta_{p}\right)$ defines the trajectory orientation angle $\theta_{p}$ in the $(x, y)$ plane and $\mathbf{V}=V \mathbf{x}_{0}$. The depinning process can be viewed as two subsequent topological electronic transitions in the vortex core which restore the anomalous spectral branch crossing the Fermi level and repair the FS. The first transition at the critical current $j_{L}=e n_{s} V_{L}$ is associated with the opening of separate segments of the FS which appear when the Doppler-shifted branch of the quasiparticle spectrum crosses the Fermi level and the minigap in the quasiparticle spectrum vanishes:

$$
V \gtrsim V_{L}=\Delta_{m}(a) / \hbar k_{F} .
$$

The latter condition coincides, in fact, with the famous Landau criterion of superfluidity [19]. The corresponding critical current density can be expressed via the depairing current density $j_{c}=e k_{F}^{2} \Delta_{0} / 3 \pi^{2} \hbar$ and CdGM spectrum:

$$
j_{L}=j_{c}\left|\varepsilon_{0}\left(k_{F} a, k_{F}\right)\right| / \Delta_{0} \leq j_{c} .
$$

Considering the zero temperature limit and assuming $a \ll \xi$ we obtain the following expressions for the critical velocity and current:

$$
V_{L}=\Delta_{0} a \ln \Lambda / \hbar k_{F} \xi, \quad j_{L}=j_{c}(a / \xi) \ln \Lambda \ll j_{c},
$$

where $\Lambda \sim \xi / a$. Taking $\tilde{\varepsilon}=0$ in Eq.(10) we find the equation defining FS segments in the $\left(\mu, \theta_{p}\right)$ plane:

$$
\varepsilon_{0}\left(\mu, k_{\perp}\right)=-\hbar k_{\perp} V \cos \theta_{p}, \quad|\mu|>\mu_{a} .
$$

These segments are shown in Fig. 2 by solid lines in the limit $a \ll \xi$ when $\varepsilon_{0}\left(\mu, k_{\perp}\right)=-\hbar \omega \mu$. The ends of the segments are joined by the lines $\mu= \pm \mu_{a}$ which correspond to the spectral branch $\varepsilon_{a}$. The direction of quasiparticle trajectory precession along the resulting closed classical orbits in the $\mu-\theta_{p}$ plane is determined by the Hamilton equation: $\hbar \partial \theta_{p} / \partial t=\partial \tilde{\varepsilon} / \partial \mu$. The formation of FS segments is obviously associated with the appearance of superconducting phase singularities outside the columnar defect.

We now proceed with the analysis of the structure of a resulting vortex state bound to the defect. For the sake of simplicity hereafter we put $k_{\perp}=k_{F}$ which is appropriate for strongly anisotropic layered material with vortex lines oriented along the anisotropy axis. One can separate two contributions to the gap function $\Delta(r, \theta)$ in the self-consistency equation: (i) the term $\Delta_{1}$ associated with the subgap states localized within the vortex core; (ii) the term $\Delta_{2}$ which is defined by the quasiparticle states with large energies $\varepsilon \gtrsim \Delta_{0}$. We introduce here the polar coordinate system $(r, \theta)$ in the plane perpendicular to the vortex axis and the origin chosen at the cylindrical defect center (see Fig. 1). At rather low temperatures the term $\Delta_{2}$ is only weakly affected by the transport current $j \ll j_{c}$ since the corresponding Doppler shift is much smaller than the superconducting gap $\Delta_{0}$. On the contrary, the first contribution to the gap is strongly modified in the presence of a transport current provided the Doppler shift exceeds the minigap $\Delta_{m}(a)$. Our further 


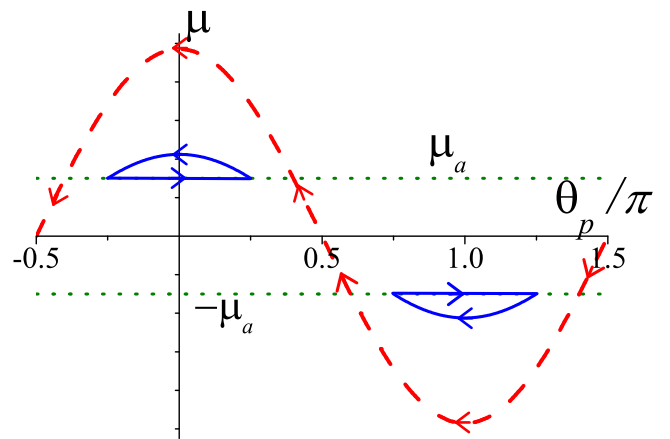

Figure 2: Fig.2 Schematic plot of Fermi surface segments in the $\left(\mu, \theta_{p}\right)$ plane for $V_{L}<V \ll V_{d}$ (solid blue line) and $V>V_{d}$ (dashed red line).

calculations of the current induced correction to the term $\Delta_{1}(r, \theta)$ are based on a semiclassical version of the selfconsistency equation (see [20]):

$$
\delta \Delta_{1}(r, \theta)=\frac{\Delta_{b}}{4 i} \int_{0}^{2 \pi} d \theta_{p} e^{i \theta_{p}}(\operatorname{sign} \tilde{\varepsilon}-\operatorname{sign} \varepsilon),
$$

where $\varepsilon(\mu)\left(\tilde{\varepsilon}\left(\mu, \theta_{p}\right)\right)$ is the subgap spectrum in the absence (presence) of transport current, $\mu=k_{F} r \sin \left(\theta_{p}-\theta\right)$, and $\Delta_{b} \sim \Delta_{0}$ is the amplitude of the gap value contribution coming from the CdGM spectral branch. Here we neglect the coordinate dependence of normalized electronand hole-like wavefunctions at the scale $r \sim a \ll \xi$ and consider the zero temperature limit. Close to the threshold velocity $V_{L}$ the spectrum $\tilde{\varepsilon}$ can be considered perturbatively in the form (1) with $k_{\perp}=k_{F}$. Note that here we restrict ourselves to the small $\mu$ limit assuming $j \ll j_{c}$. For small superfluid velocities $V \leq V_{L}$, the gap function $\Delta_{1}=\Delta_{b} e^{i \theta}$ is undisturbed $\left(\delta \Delta_{1}=0\right)$. Otherwise, if $V>V_{L}$, the Doppler shift results in qualitative changes in the gap function distribution. A resulting piecewise regular gap function

$$
\Delta_{1}(r, \theta)=\left|\Delta_{1}\right| \exp (i \phi)=\Delta_{b} \mathrm{e}^{i \theta}+\delta \Delta_{1}(r, \theta)
$$

is defined in a set of regions shown in Fig. 3:

$$
\Delta_{1}(r, \theta)=\Delta_{b} \mathrm{e}^{i \theta} \begin{cases}1, & \text { in } A \text { or } C, \\ 1-2 \sqrt{r^{2}-a^{2}} / r, & \text { in } B, \\ 1-\mathrm{e}^{i \alpha_{r}}+\mathrm{e}^{-i \alpha_{0}}, & \text { in } D, \\ 1-\mathrm{e}^{-i \alpha_{r}}+\mathrm{e}^{-i \alpha_{0}}, & \text { in } E,\end{cases}
$$

$$
\text { where } \sin \alpha_{r}=\frac{a}{r}, \quad \sin \alpha_{0}=\frac{r_{0} \cos \theta}{\sqrt{r^{2}+2 r_{0} r \sin \theta+r_{0}^{2}}},
$$

and $r_{0}=V / \omega$. By dashed lines in Fig. 3 we also show schematically the distribution of the superconducting phase gradient $\nabla \phi$ around the defect. One can clearly

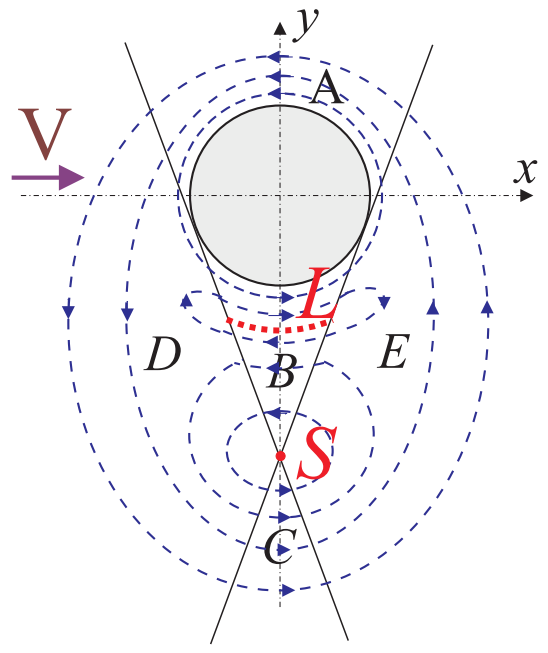

Figure 3: Fig.3 Schematic distribution of the superconducting phase gradient $\nabla \phi$ (dashed lines) for a bound vortex configuration.

observe strong changes in topology of the phase $\phi$ distribution caused by the superflow: there appear a phase singularity at the point $S\left(x=0, y=-r_{0}\right)$ and the line $L(r=2 a / \sqrt{3})$ of zeros of the superconducting order parameter $\Delta_{1}$ in the region $B$ (see Fig.3). The singularity at the point $S$ has the same vorticity as the initial vortex captured by the defect. With an increase in the superfiuid velocity $V$ this singularity moves away from the defect. One can see that the modification of the phase $\phi$ distribution shown in Fig. 3 corresponds to the formation of a bound vortex-antivortex pair. Note that the vortex and antivortex positions found here coincide with the positions of the phase singularities of a total gap function since the amplitude of the second part of the gap function $\Delta_{2}$ is rather small: $\left|\Delta_{2}\left(r \sim r_{0}\right)\right| \sim \Delta_{0} r_{0} / \xi \ll \Delta_{b}$.

Certainly well above the threshold velocity $V_{L}$ the gap profile and stable vortex configuration should be determined from the self-consistent procedure taking account of contribution of delocalized excitations. It is natural to expect that the instability of the bound vortex state and appearance of a freely moving vortex should be accompanied by the formation of a continuous anomalous branch connecting the energies below $-\Delta_{0}$ and above $+\Delta_{0}$. It is this branch which provides necessary conditions for spectral flow through the energy gap and, thus, for energy dissipation during the vortex escape from the defect. The obstacles on the way of quasiparticle flow through the gap could be removed provided the FS segments would merge and reconnect to form a cosine curve $\mu\left(\theta_{p}\right)$ typical for a free vortex with a continuous anomalous spectral branch (compare solid and dash lines in Fig. 2). This transformation causes topological changes in FS geometry because new cosine FS is open and can not be contracted to a 
point by continuous transformations. Correspondingly, one can suggest three obvious scenarios of destruction of the bound vortex configuration: (i) first, the FS segments can merge forming an open FS due to quantum mechanical tunneling; (ii) second, the spectral flow through the energy gap can occur due to the impurity scattering between different Fermi surface segments; (iii) third, the free vortex can be formed when the Doppler shift reaches the gap value, i.e., the current density approaches the depairing one. The first scenario can be realized due to the Landau-Zener tunneling between classical orbits and can be understood within the quantum mechanical picture based on the commutation relation for canonically conjugated variables $\mu$ and $\theta_{p}:\left[\hat{\mu}, \hat{\theta}_{p}\right]=-i$. Here we use square brackets to denote a commutator of two operators. The efficiency of this tunneling is determined by the ratio of the distance $2 \mu_{a}$ between the orbits to the angular momentum uncertainty $\Delta \mu$. The latter value can be estimated from the uncertainty principle $\triangle \mu \triangle \theta_{p} \sim 1$ and the equation $\mu=k_{\perp} V \cos \theta_{p} / \omega$ describing the classical orbit for small $\mu$ values. Considering the trajectories with $\theta_{p}$ close to $\pm \pi / 2$ we find: $\Delta \mu=k_{\perp} V \Delta \theta_{p} / \omega$ and $\Delta \mu \sim \sqrt{k_{\perp} V / \omega}$. Thus, Landau-Zener tunneling results in the merging of two FS segments provided the condition $\mu_{a}<\sqrt{k_{\perp} V / \omega}$ is fulfilled. This second transition in the spectrum accompanied by the change in the Fermi surface topology occurs at the critical velocity and current given by the relations:

$$
V_{d}=k_{F} \omega a^{2}, j_{d}=j_{c}\left(k_{F} a^{2} / \xi\right) \ln \Lambda=k_{F} a j_{L} \gg j_{L} .
$$

For $j>j_{d}$ the FS is described by the Eq. 5 for all $\mu$ values (see dashed line in Fig. 2) and corresponds to a Doppler-shifted spectrum of a free vortex: $\tilde{\varepsilon}=$ $-\hbar \omega \mu+\hbar k_{F} V \cos \theta_{p}$ for $|\mu| \ll k_{F} \xi$. The continuous path for the quasiparticle spectral flow from the energies below $-\Delta_{0}$ to the ones above $+\Delta_{0}$ is now restored providing conditions for a dissipative vortex motion.

Second scenario of the recovering such continuous path through the gap is associated with impurity scattering. The scattering rate $1 / \tau_{s}$ between the quasiparticle states at different FS segments shown in Fig. 2 should be reduced compared to the impurity scattering rate $1 / \tau$ in a normal metal due to the small size $\delta \theta_{p}$ of FS segments in the phase plane $\mu-\theta_{p}: 1 / \tau_{s} \sim \delta \theta_{p} / \tau$, where $\cos \left(\delta \theta_{p} / 2\right)=\omega a / V$. Provided the scattering rate $1 / \tau_{s}$ becomes comparable with the minigap $\Delta_{m}=k_{F} a \hbar \omega$ for a pinned vortex the FS segments can be no more considered as isolated ones. This condition imposes a restriction on the size of the isolated FS segments $\delta \theta_{p}<\delta \theta_{\max }=\gamma \Delta_{m} \tau / \hbar<\pi$ and gives us an estimate for the upper critical velocity and current which destroy the bound vortex state:

$$
V_{d}=\frac{V_{L}}{\cos \left(\delta \theta_{\max } / 2\right)}, j_{d}=\frac{j_{L}}{\cos \left(\delta \theta_{\max } / 2\right)},
$$

where the constant $\gamma$ is of the order unity. Note that
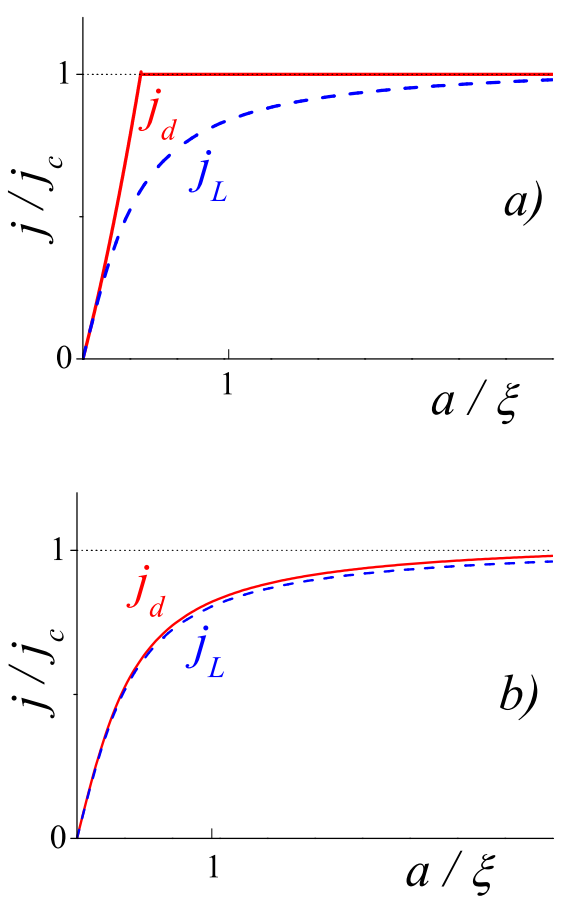

Figure 4: Fig.4 Schematic dependencies of critical current densities vs the defect radius $a$ for clean (a) and dirty (b) limits.

in the limit $\gamma \Delta_{m} \tau / \hbar>\pi$ the impurity scattering effect is negligible and the depinning current density is given by the Eq. 8 Let us also emphasize that the critical current of vortex depinning can not exceed the depairing one. Thus, Eqs. (8, 9) are valid only for $j_{d}<j_{c}$, i.e., for rather small size of the defect. In the clean limit, e.g., this restriction on the size reads: $a \leq \sqrt{\xi / k_{F}} \ll$ $\xi$. For larger defect radii the depinning current density should saturate at the $j_{c}$ value. The resulting schematic dependencies of critical current densities vs the defect radius $a$ are shown in Fig. 4 for clean (a) and dirty (b) limits. One can see that the unusual bound vortex state near the defect can be observed only for rather clean samples with $\Delta_{0} \tau / \hbar \gg 1$. In the dirty case for $\Delta_{0} \tau / \hbar \leq$ 1 both critical current densities $j_{L}$ and $j_{d}$ coincide. The depinning current estimate in this limit can be also found following the approach developed in Refs. 6, 7] for the pinning on impurities with small scattering cross section.

To sum up, we have developed a microscopic description of the two-stage scenario of vortex depinning from small size columnar defect and predict the formation of stable vortex - antivortex configurations bound to the defect at intermediate transport current densities. The lower critical current density corresponds to the formation of vortex -antivortex state and should determine rf nonlinear response of vortex system at rather small 
transport current amplitudes. The upper critical current density $j_{d}$ corresponds to the destruction of the bound vortex configuration around the defect and should determine the depinning transition at dc currents.

The authors are thankful to Mike Silaev for many helpful discussions. This work was supported, in part, by the Russian Foundation for Basic Research, Russian Agency of Education under the Federal Program "Scientific and educational personnel of innovative Russia in 2009-2013", and European IRSES program SIMTECH (contract n.246937).

[1] G. Blatter, M. V. Feigel'man, V. B. Geshkenbein, A. I. Larkin, V. M. Vinokur, Rev. Mod. Phys. 66, 1125 (1994).

[2] G. S. Mkrtchyan and V. V. Shmidt, Zh. Eksp. Teor. Fiz. 61, 367 (1971) [Sov. Phys. JETP 34, 195 (1972]].

[3] A. Buzdin and D. Feinberg, Physica C 256, 303 (1996).

[4] A. Buzdin and M. Daumens, Physica C 294, 257 (1998).

[5] H. Nordborg and V. M. Vinokur, Phys. Rev. B 62, 12408 (2000).

[6] E. V. Thuneberg, J. Kurkijarvi, and D. Rainer, Phys. Rev. Lett. 48, 1853 (1982); Phys. Rev. B 29, 3913 (1984).

[7] E. V. Thuneberg, J. of Low Temp. Physics 57, 415
(1984).

[8] C. Caroli, P. G. de Gennes, J. Matricon, Phys. Lett. 9, 307 (1964).

[9] L. Kramer and W. Pesch, Z. Physik 269, 59 (1974).

[10] N. B. Kopnin, Theory of Nonequilibrium Superconductivity (Clarendon Press, Oxford, 2001).

[11] G. E. Volovik, The Universe in a Helium Droplet, Clarendon Press, Oxford, 2003.

[12] I. M. Lifshits, Zh. Eksp. Teor. Fiz. 38, 1569 (1960) [Sov. Phys. JETP 11, 1130 (1960)].

[13] Y. M. Blanter, M. I. Kaganov, A. V. Pantsulaya, A. A. Varlamov, Phys. Reports 245, 159 (1994).

[14] G. E. Volovik, Pis'ma Zh. Eksp. Teor. Fiz. 49, 343 (1989) [JETP Lett. 49, 391 (1989)].

[15] A. S. Mel'nikov, D. A. Ryzhov, and M. A. Silaev, Phys. Rev. B 78, 064513 (2008).

[16] A. S. Mel'nikov and M. A. Silaev, Pis'ma Zh. Eksp. Teor. Fiz. 83, 675 (2006) [JETP Lett. 83, 578 (2006)].

[17] A. I. Larkin and Yu. N. Ovchinnikov, Phys. Rev. B 57, 5457 (1998).

[18] A. S. Mel'nikov, A. V. Samokhvalov, M. N. Zubarev, Phys. Rev. B 79, 134529 (2009).

[19] L. D. Landau and E. M. Lifshitz, Statistical physics. part 2, Pergamon, New York, 1980.

[20] G. E. Volovik, Pis'ma Zh. Eksp. Teor. Fiz. 58, 444 (1993) [JETP Lett. 58, 455 (1993)]. 